\section{UNA DEMOCRATIZACIÓN \\ TAMBALEANTE. ESCOLARIZACIÓN Y COHESIÓN SOCIAL EN CHILE, 1920-1960}

A STUMBLING DEMOCRATIZATION. SCHOOLING

AND SOCIAL COHESION IN CHILE, 1920-1960

\section{FRANCISCA RENGIFO STREETER •}

Centro de Estudios de Historia Política, Universidad

Adolfo Ibáñez (Chile).

E-mail: francisca.rengifo@uai.cl

\section{Resumo}

Este artículo aborda la relación entre escolarización y cohesión social en Chile durante la masificación de la educación primaria promovida por la obligatoriedad escolar establecida en 1920.

carácter extensivo del proceso interpeló el sentido socialmente inclusivo de la escuela, lo que evidenció una profunda fractura nacional encarnada en miles de niños desnutridos. Para comprender las tensiones y mutuas transformaciones entre la escuela constructora de un común denominador social y unas expectativas de inclusión expresadas en el discurso democratizador del período, se analizan la cobertura educacional, la deserción estudiantil y el auxilio escolar. Los resultados constatan que, si bien diversos actores diagnosticaron el subdesarrollo biocognitivo entre la población infantil, la política educacional fue desigualmente inclusiva y asumió que el sistema de seguridad social enfrentaría este problema indirectamente.

\section{Registro bibliográfico}

RENGIFO STREETER, FRANCISCA «Una democratización tambaleante. Escolarización y cohesión social en Chile, 1920-1960», en: ESTUDIOS SOCIALES, revista universitaria semestral, año XXIX, $n^{\circ} 57$, Santa Fe, Argentina, Universidad Nacional del Litoral, julio-diciembre, 2019, pp. 161-183.

\section{Abstract}

This article addresses the relationship between schooling and social cohesion in Chile during the mass schooling process that began with the compulsory school established in 1920. The extensive nature of this process intervened in the socially inclusive sense of education, evidencing the national fracture embodied in thousands of malnourished children. To understand the tensions and mutual transformations between the school as a builder of a common social denominator and the expectations of cohesive inclusion expressed in the democratizing discourse of the period, it analyses the coverage, the desertion and the school assistance policy. The results show that, although several actors diagnosed the physicalcognitive underdevelopment suffered by the child population, the educational policy was unequally inclusive, and it assumed that the social security system would face this problem indirectly.

\section{Descriptores · Describers}

Educación pública / Cohesión social / Escolarización / Pobreza / Estado de bienestar

Public education / Social cohesion / Schooling / Poverty / Welfare State

Recibido: 19/03/2019 Aceptado: 10/07/2019 


\section{INTRODUCCIÓN ${ }^{1}$}

La educación ha sido estrechamente relacionada a la idea de cohesión social por su capacidad de crear y transformar lo común. Este supuesto atribuye a los sistemas nacionales de educación una capacidad de crear lazos sociales que promueven formas de pertenecer a la sociedad, incentivando un sentimiento de grupo a partir de una experiencia escolar compartida. La escuela, como sistema de enseñanza formal establecido por los estados nacionales, devino en un espacio de interacción y de procesos de integración social en el cual las personas experimentan y aprenden a pensarse como partes de un colectivo mayor (COX Y SCHWARTZMAN, 2009). Esta cualidad primordial atribuida a la escuela como transformadora de lo social es el correlato político del concepto sociológico de cohesión referido al "pegamento social», a aquello que hace que una sociedad permanezca unida (DURKheim, 1967). Por tanto, siendo la educación una construcción política, la escuela es considerada como un componente fundamental del conjunto de normas e instituciones compartidas y también del imaginario social y simbólico que asocia unas representaciones de lo común con unos significados cohesionadores respecto de una sociedad específica (CASTORIADIS, I993; FULLER y RUBINSON, I992).

Este supuesto se apoya en unos sistemas nacionales de educación que tardaron décadas en expandirse. Por consiguiente, para comprender cómo la escuela construye lo común es ineludible atender al proceso de escolarización masiva del cual emergieron aspiraciones a una democracia más inclusiva, revelando al mismo tiempo sus propias formas de exclusión. Tanto la institucionalización de la escuela obligatoria entre fines del siglo xIx y comienzos del xx, como su consiguiente expansión, son dos fenómenos que contribuyen a explicar la actual prioridad otorgada a la cohesión social como democratizadora y su formulación en política pública. Este entrelazamiento anuda la noción normativa de cohesión social como una capacidad de las sociedades democráticas estables — porque ellas establecen equilibrios y generan dinámicas sociales que evitan los riesgos de fragmentación social y crisis - con la política educacional como un vehículo certero para promoverla y reforzarla (BARBA y COHEN, 2OII; CUELLAR, 2OIO; TIRONI, MELLER y PEÑA, 2008).

1] Este artículo es parte de los resultados del proyecto «Formas y representaciones de la cohesión social. Una perspectiva histórica a partir de experiencias de sociabilidad sudamericanas, 1870-1960" HAR 2015-65564. 
En Chile, como en otros países latinoamericanos, la institucionalización de una educación gratuita, universal y obligatoria, fue un fenómeno que no correspondió temporalmente a su masificación. Este proceso adquirió rápido crecimiento en la segunda mitad del siglo $\mathrm{xx}^{2}$. Precisamente por su carácter extensivo, este evidenció nuevas dinámicas excluyentes representadas por los elevados índices de deserción escolar y analfabetismo comunes a la región. Para comprender esas tensiones y las mutuas transformaciones entre la escuela constructora de un común denominador social y unas expectativas de inclusión cohesionadora expresadas en el discurso democratizador, este artículo analiza el proceso de escolarización chileno durante el período de masificación de la educación primaria a partir de la obligatoriedad escolar establecida en I920. Los ritmos del proceso revelan la precariedad sociocultural del proyecto político y del modelo de desarrollo nacional, lo cual denota una profunda fractura nacional encarnada en miles de niños desnutridos. Por un lado, el desencuentro entre la compulsión escolar y la realidad social que indican las estadísticas desagregadas de matrícula y de deserción escolar constatan que la escolarización fue un proceso desigualmente inclusivo; por otro, la posibilidad científica de conocer y ponderar la dimensión biológica en la cual ocurre el aprendizaje redefinió el sentido cohesionador de la educación en nuevos términos democráticos. En consecuencia, este artículo plantea que la escolarización nacional revela los pies de barro de la democracia chilena, identificando un hiato entre la educación como derecho social y la desigualdad estructural del país, el cual es crucial para pensar la cohesión social.

El proceso de escolarización estuvo enmarcado por dos momentos críticos. A comienzos del siglo xx, la cuestión social y la crisis político-económica experimentadas impulsaron reformas estatales y el establecimiento de una serie de leyes llamadas sociales que redefinieron las relaciones entre el Estado y la sociedad (RENGIFO, 20I7; ROSEMBLATT, 200I). Cinco décadas más tarde, cuando la educación primaria adquirió un perfil masivo a fines de los 6o, nuevos procesos de quiebre

2] En los países latinoamericanos la obligación escolar fue establecida a comienzos del siglo XX en una sociedad escasamente escolarizada y la masificación de la educación primaria fue un fenómeno impulsado por los estados nacionales (PARRADO, 1998; TORRES y PUIGGROS, 1995). La aproximación institucional subraya la naturaleza ideológica y también transnacional de la educación masiva, explicando este fenómeno dentro del sistema mundial (RAMÍREZ y BOLI, 1987; MEYER, RAMÍREZ y NUHOGLU, 1992; BENAVOT, 1997). 
social y político mostraron que los esfuerzos educacionales no respondieron a una estrategia por una sociedad menos segregada y más cohesiva. Si bien universalizar la escuela fue un objetivo políticamente transversal, reiterado por los diversos gobiernos del período y asociado a la difusión de unos ideales comunitarios expresados como democracia social, este fue solo parcialmente efectivo. La escuela fue un instrumento de cohesión social, a la vez que un registro de su éxito o fracaso. Así lo mostró la figura gráfica correspondiente a la cobertura educacional en estas décadas. Las estadísticas oficiales dibujaron una base social ancha pero educacionalmente delgada, pues los escolares no cursaban más de dos años de enseñanza. Es decir, la extensión de la matrícula no correspondió a una expansión de la escolaridad.

A partir de este piso empírico, el presente análisis argumenta que, aunque el diagnóstico de la crisis educacional chilena identificó que integrar los niños a la escuela requería involucrarlos en el proceso de enseñanza, los modos de implementar esa política educacional no priorizaron la escolarización, sino que más bien frustraron el objetivo de universalización establecido por la ley³. El mayor obstáculo para el aprendizaje escolar era el hambre que padecía la inmensa mayoría de la población infantil. Los nuevos profesionales médicos requeridos por las políticas de bienestar demostraron que masificar la educación era un proceso frustrado por generaciones de niños desnutridos y dañados en su desarrollo vital. A partir de este diagnóstico, las autoridades políticas y la opinión pública coincidieron en que revertir los condicionamientos fisiológicos que impedían la educación requería organizar un sistema de auxilio escolar en alimentación y salud. Sin embargo, esos proyectos asumieron que la incipiente seguridad social indirectamente lo resolvería y dispusieron un débil servicio de protección social.

Desde la arista biológica del problema educacional, la cohesión social puede observarse desde la vereda opuesta a la experiencia escolar compartida, como un fenómeno de exclusión (BEHAGG, 1997). Asimismo, revela la disyuntiva de responsabilizar a la escuela como política de cohesión social y no articular unas políticas basales para hacerla posible. Por ello, abordar la relación entre educación y cohesión social implica examinar dicho proceso de escolarización como una política que buscó anclar, proveyendo de asidero concreto, unas formas y representaciones de vínculos sociales inclusivos y estructura el análisis en tres secciones. La primera, de carácter cuantitativo, analiza la cobertura educacional primaria, identificando las

3] Matizando otras interpretaciones centradas en el Estado docente (ILLANES, 1990; EGAÑA, 1995). 
dinámicas internas a este ciclo expansivo entre los años 1920 y 1960 que definieron su carácter desigual en cuanto a las oportunidades educacionales de una población escolar que no vio asegurada una enseñanza formal completa. Mediante la reconstrucción del diagnóstico contemporáneo sobre el problema de rezago escolar, la segunda sección constata la desigualdad estructural de la sociedad chilena. La barrera biológica, explicada por los especialistas médicos, pedagogos y economistas como un subdesarrollo físico cognitivo de los niños, ensombreció las expectativas del discurso político e interpeló al sentido socialmente inclusivo de la educación. La tercera sección da cuenta del giro democratizador expresado por la universalización del derecho social a la educación a partir de la obligatoriedad escolar. Las expectativas políticas de ampliación representativa también incorporaron nuevos derechos de sectores cada vez mayores de la sociedad y perfilaron así una segunda fase democratizadora (MARSHALL, I964; O'DONNELL, I999; HUNTINGTON, I99I; ROSANVALLON, 2OII y 1995). Estas aspiraciones no eran la universalización del voto, establecida en la Ley Electoral de I874, reafirmada por el voto secreto en I884, y reformada en I888 como el derecho de todos los chilenos — varones- con 2I años de edad, que supieran leer y escribir. El desafío era la participación efectiva de amplios sectores de la población hasta entonces socialmente marginados ${ }^{4}$. La construcción social de la democracia a través de la educación estuvo constreñida por las crisis económica, política y social, compitiendo la educación con otros programas sociales por los restringidos recursos y relegando a un plano secundario las reformas a la escuela (LABARCA, I939; HAMUY, 1960). La necesidad de un programa de bienestar escolar para contrarrestar la pobreza quedó supeditada a un sistema de seguridad social laboralmente segmentado y no desarrolló un programa global de asistencia. La imposibilidad de reformas fue síntoma del agotamiento del sistema político en un contexto de bajo crecimiento económico dependiente de las exportaciones y deprimido por la inflación endémica. Los esfuerzos de democratización mediante la escuela también siguieron un patrón segmentado correspondiente a los intereses del sistema político por cooptar sectores estratégicos de la población urbana, como los trabajadores de la minería y metalurgia y los empleados, en desmedro de amplios sectores sociales como el rural y los pobres urbanos.

4] A partir de la Constitución de 1925, el régimen electoral adquirió unas instituciones autónomas con mayor independencia de la contienda política y abrió ciclos de democratización del voto, ampliando fuertemente la participación popular (VALENZUELA, 1985; URZÚA, 1992; TORRES, 2014). 
La trayectoria descrita sugiere un ideario de cohesión social dentro del paradigma democrático y de bienestar, que giró desde una concepción popular-universalista asociada a los gobiernos radicales entre las décadas de 1930 y 1940 hacia una desarrollista, estimulada por actores internacionales y que entendió a la educación como inversión pública en capital humano. La pertinencia de estudiar cuantitativa, social y políticamente dichos procesos radica en que factores considerados causales por las evaluaciones técnicas que concentran unos parámetros de cohesión social se tornan variables interrelacionadas que permiten hacer inteligible la relación diacrónica entre cohesión social y educación. Si bien la relación entre educación y cohesión social ha sido históricamente construida, el campo de análisis ha concentrado la atención sobre indicadores relativos a la mayor o menor capacidad de la escuela por promover un sentido social de cohesión y/o modelos normativos que recomiendan reformas institucionales y políticas. En cambio, abordar la cohesión social como un fenómeno histórico permite recuperar la dimensión temporal de dicha relación, explicitar sus expectativas concretas y nutrir así dicho concepto de contenido específico. En tales procesos de negociación, formulación e implementación de la política educacional, habría expectativas de cohesión social que articulan el sentido específico mediante el cual los actores involucrados comprendieron estos procesos 5 . De este modo, es posible evitar el riesgo de que la familiaridad del término cohesión social dé por evidente aquello que hay que explicar y, a su vez, rescatar el potencial huerístico que el concepto posee para explorar el proceso de escolarización como una fuerza de cambio social.

\section{LA ESCUELA, COMÚN DENOMINADOR SOCIAL}

La educación como institución cohesiva es inseparable del proceso de escolarización nacional. Su carácter universal como derecho individual fue formalizado en la Ley de Instrucción Primaria Obligatoria de 1920, que pretendía conjurar la crisis educacional representada por los fenómenos de analfabetismo y ausentismo escolar. La formalización de la escuela pública en la década de I880 había agilizado

5] Desde la historia conceptual, el carácter procesual de un concepto indaga por cuáles significados de una idea poseen unos actores atribuyéndole sentido a unos acontecimientos, preparando y concretando un fenómeno determinado que, a su vez, resignifican el concepto (KOSELLECK, 2012). 
su extensión territorial con apoyo de los recursos fiscales, pero esta red nacional de escuelas — todavía insuficiente - tenía sus aulas medio vacías (sOFIER, 2009; PONCE DE LEÓN, 2OIO). El número de establecimientos se triplicó, aumentó de I248 escuelas en I895 a 3148 en I920, pero la inasistencia entre los alumnos persistía tenazmente. El porcentaje de escolares ausentes era elevadísimo y se había estancado en dichos años oscilando entre un $62,7 \%$ y el $60,8 \%{ }^{6}$. Para el destacado educador e impulsor de la obligatoriedad escolar, Darío Salas, esta ley era un proyecto de comunidad nacional. Era impensable una sociedad cohesiva en la cual los niños analfabetos representaban una lanza que atravesaba el país de norte a sur. ${ }^{7}$ Implementar una educación básica común requería garantizar el derecho a un mínimo de educación definido por la ley en seis años de enseñanza para la población urbana y cuatro para la rural. Pero este sentido universal, expresado como promesa política de los gobiernos de Arturo Alessandri (I920-I925 y 1932-1938), Carlos Ibáńez (1927-193I y 1952-1958) y los radicales iniciados por Pedro Aguirre (1938-1944, I944-1952), no fue evidente al contrastarlo con el ritmo ralentizado que tuvo la escolarización en el período.

Entre 1930 y 1960 la escuela se hizo una experiencia transversal a todos los sectores sociales, tanto en el campo como en los poblados. Haber recibido una enseñanza formal —aunque de corta duración — llegó a ser un común denominador de la sociedad chilena. La mayoría había pasado por la escuela y sabía leer y escribir. La cobertura educacional primaria respecto de los niños y niñas entre 5 y I4 años de edad aumentó de un 4I,I \% en 1920 al 6I,4 \% en I960, continuando la tendencia extensiva anterior (GRASSAU Y ORELLANA, I959: 64; PONCE DE LEÓN, 20I8). Pero las estadísticas aún constataban que un $40 \%$ de la población en edad escolar recibía un servicio educativo nulo o de hasta dos años de primaria. Las cifras de la cobertura primaria reflejaron una continuidad ascendente desde fines del siglo XIX, sobrepasando a la mayoría de dicha población en I930 (52,8\%). En tanto, el balance fue insatisfactorio en términos de escolaridad. Desde una perspectiva endógena al sistema escolar, este crecimiento significó que un mayor número de alumnado recibió enseñanza primaria, pero la proporción de niños que

6] Cifras calculadas a partir del Anuario Estadístico de la República de los años 1890 a 1920 y Censos de población de 1895, 1907 y 1920.

7] Proyecto de obligatoriedad escolar presentado al Congreso Nacional sobre la base del formulado por Darío Salas (1917). 
no ingresaban a la escuela no disminuía e incluso aumentó. Los niños matriculados asistieron proporcionalmente en mayor número a la escuela —alcanzaron un $83 \%$ en 1956- pero el porcentaje de inescolares en la cohorte poblacional entre 6 y I2 años de edad había crecido en tan solo una década de 23,8 \% a 28,2 \%, como lo registró el censo nacional de 1940 (GRASSAU y ORELLANA, I959: 15-16) ${ }^{8}$.

Estos desajustes del crecimiento educacional revelan que el proceso de escolarización no fue comprehensivo social ni espacialmente. El desglose estadístico de la matrícula constata que de cien niños matriculados en el primer curso, menos de un quinto estaban registrados en el sexto. Es decir, más de la mitad de la población que ingresaba a la escuela no completaba la primaria. En la década de I960, dicha proporción había aumentado a casi un tercio, pero todavía la mayoría de los estudiantes no alcanzaba la meta. La persistencia de la deserción escolar devela que la extensión de la cobertura no fue equivalente a la expansión de la escolaridad. Como resultado colateral, la escuela obligatoria tampoco aseguraba la continuidad de los estudios hacia los siguientes niveles, reforzando la estructura piramidal de un sistema dividido entre los compartimentos primario, secundario y superior. El censo nacional de 1952 hizo la radiografía social: de cada mil estudiantes en el país, 806 estaban matriculados en la primaria, II9 en el liceo, 59 en las escuelas especiales y solo i6 en las universidades. Este diseño segmentado, denunciaron las autoridades políticas y los especialistas educacionales, reproducía la desigualdad social del país y contradecía las aspiraciones de la política pública por concretar la enseñanza primaria completa y crear posibilidades de continuidad hacia la secundaria y técnica-superior. Entre 1960 y 1964, las cifras de matrícula mostraron el patrón desigual adquirido por el proceso expansivo: alcanzó al 94 \% de la población en edad escolar, pero solo un $63 \%$ de los matriculados egresaban.

Institucionalmente, la oferta educacional diferenció entre la población rural y la urbana, incidiendo en esta desigual expansión, ya que refleja la dimensión espacial del ideario cohesionador contenido en la política educacional. La reglamentación definitiva de la obligatoriedad escolar y su implementación fueron elementos centrales de la refundación del Ministerio de Educación en los años 1927-1929, como parte del proceso centralizador y burocratización del sistema educacional $-\mathrm{y}$ del

8] Cálculos estimados a partir de los censos de la república de Chile de 1907, 1920 y 1930, y de los Anuarios Estadísticos del período. 
Estado en general - a partir del gobierno del general Ibáñez y reimpulsada tras la crisis económica del '3o que había paralizado en la práctica su efectividad. En dos décadas desde la promulgación de la obligatoriedad, los servicios educacionales resintieron un país en crisis y las reorganizaciones caóticas del ministerio. Las reformas por dotar de unidad al sistema educacional comenzaron a ensayarse en la década de 1940 y enfrentaron un entramado institucional excesivamente departamental, así como un sector docente políticamente poderoso, por lo cual tales esfuerzos fueron estériles.

La deficiencia institucional iba de la mano de una crisis de la infraestructura en educación primaria. Por un lado, el sistema no ofrecía una enseñanza completa para todos los niños del país, limitando la obligatoriedad en las zonas rurales. Esta disposición legal atendía a que la gran mayoría de las escuelas no contaba con un plan de estudios completo. De las 3162 existentes, un 65,6 \% no ofrecía ni siquiera los primeros cuatro grados de estudios. Por otro lado, tampoco había suficientes escuelas para satisfacer la demanda educacional ni presupuesto de la nación para invertir más en ellas. A pesar del agudo movimiento migratorio hacia los grandes centros urbanos, el déficit institucional significó la menor posibilidad de completar la enseñanza primaria para los habitantes de las zonas rurales. Entre las unidades político-administrativas en las cuales se dividía el país, aquellas que concentraban la mayor proporción de población rural tenían en funcionamiento a menos de un $15 \%$ de sus escuelas con plan de estudios completo. A nivel nacional, dicha desproporción significó que los escolares en dos tercios del total de escuelas enfrentaron un techo educacional no superior a tres ańos de enseńanza.

Gráficamente, la curva ascendente de la cobertura primaria ocultó su desequilibrio interno. Este desajuste fue sospechado por el refundado Ministerio de Educación, el cual realizó un censo educacional para diagnosticar su política de escolarización. Levantado en I933, el censo registró a los niños inscritos en cada curso del nivel primario e identificó el patrón rezagado que había adquirido el proceso de escolarización. Las edades de los matriculados en un curso rebasaban ostensiblemente los límites etarios considerados normales para aquel nivel. Si en el primer curso debían estar nińos entre 6 y 7 años de edad, el $79 \%$ de ellos era mayor; y si en tercero sus edades no debían ser superiores a io años, el 85,5 $\%$ también era mayor. Esta falta de correspondencia entre la edad biológica y la pedagógicamente adecuada evidenciaba un retraso escolar que era sintomático a los datos de alfabetización que mostraban un nivel cultural muy insatisfactorio. 
Socialmente, estos cálculos de deserción y rezago escolar insinuaron una profunda fractura nacional cuyas causas, denunciaron las autoridades educacionales, políticas y grupos de profesionales — principalmente médicos - vinculados al Estado, no estaban en la escuela sino fuera de ella. Constatarlas fue el comienzo de una abundante tarea investigativa y de estudios especializados que diagnosticaron a la desnutrición como el factor determinante del problema. En palabras de la emblemática educadora Amanda Labarca a comienzos de los años i940, la instrucción básica sufría el abandono por parte de un sistema educacional que había privilegiado a las elites de los liceos y a la enseñanza universitaria. La nación necesitaba de la cohesión de todas sus fuerzas, pero esa unidad era imposible «si no nos hacemos solidarios del destino de nuestros estratos olvidados» (LABARCA, I939 y I943: 56 y 8I). Las deficientes condiciones en que se desarrollaba la educación, o sea la popular, mantenían la adversa situación de los pobres y, en tales circunstancias, la escuela era inconcebible como vía de mejoramiento social. El sistema escolar debía proteger la vida del niño, demandaba la educadora, porque ese individuo sería un elemento aglutinador indispensable en una sociedad demasiado laxa. Nuestra centenaria trayectoria educacional representaba un peligro si se consideraba al sistema como un medio para mantener logros ya conseguidos y no como posible factor de desarrollo nacional. Esta fue la alerta dada por la comisión oficial para el planeamiento educacional y que recogió posteriormente la reforma realizada por el gobierno de Frei Montalva (1964-1970) con el fin de reimpulsar la escolarización. Además de crear más aulas, el objetivo de la reforma era impactar en la igualdad de oportunidades, concretando los principios de una educación libre y equitativa del Programa de Reforma en Libertad del Partido de la Democracia Cristiana por primera vez en el poder. Un mecanismo crucial para enfrentar la crisis social era una educación pública que formara integralmente al individuo como motor del progreso y trabajador eficaz a la productividad del país?.

9] Ministerio de Educación, Bases generales para el planeamiento integral de la educación chilena. 


\section{EL QUIEBRE SOCIAL}

La crisis educacional del periodo reveló una profunda fractura nacional que fue formulada en términos de subdesarrollo biológico. La reformulación del problema significó desplazarlo del ámbito educacional al de salud o, más bien, reconocer el carácter multisectorial de este. La educación representaba una fuerza cohesionadora que carecía de piso social donde enraizarse pues la pobreza, que había sido un fenómeno omnipresente en el siglo anterior, permanecía enquistada como la principal barrera a la expansión de la escolaridad. El hambre era un fenómeno persistente entre generaciones de chilenos perturbados en su capacidad cognitiva. Esta conclusión implicó entender a la pobreza en su contingencia biológica, recurriendo a innovadoras categorías médicas y formas de medición internacionalmente estandarizada que hacían posible un nuevo conocimiento social. Ponderada tanto en las calorías diarias necesarias para el desarrollo vital como en medidas antropométricas, la desnutrición desvanecía todo esfuerzo educacional, desafiando el principio democrático de la dignidad humana.

La cruda evidencia que acumularon los estudios sociomédicos, así como en otros países latinoamericanos redefinió la discusión pública sobre la pobreza ahora como desigualdad social (sCHTEINGART, I935; POBLETE, I942). La primera encuesta de envergadura por su metodología y alcance nacional fue realizada en 1935 por la Sección de Higiene de la Sociedad de las Naciones en colaboración con el gobierno de Chile (Dragoni y burnet, 1939). Más de un tercio de la población (37,2\%) sufría un déficit alimentario grave, menor a una ingesta de dos mil calorías diarias, y otro décimo (I2 \%) estaba subalimentado. Este déficit presentó además una desigual distribución entre la población infantil: las curvas de desarrollo presentadas por los niños de hogares pobres eran inferiores a los de aquellos con mayores recursos. Tres décadas más tarde, esta radiografía social no había experimentado mayores alteraciones. En 1960, los niños pobres aún no alcanzaban los indicadores fisiológicos que habían tenido sus pares socioeconómicamente más acomodados en 1908: les faltaba en promedio diez kilos de peso y diez centímetros de altura (ARIZTíA, 1967). Esta desventaja también persistía entre los jóvenes que asistían a las escuelas y los que iban al liceo: el $60 \%$ de los primeros tenía una estatura y peso subnormal (Junta Nacional de Auxilio Escolar, Memoria de 1958: 8).

Paralelamente, el continuo examen médico a los estudiantes sistematizado por el Servicio Nacional de Salud creado en 1952, indagó en esa relación física-cognitiva vinculando el estado de subnutrición infantil al fracaso escolar. La consecuen- 
cia de una dieta reducida en calorías y carente de proteínas, concluyeron estas estimaciones, era que las deficiencias de peso y altura correspondían también a la pérdida del volumen o de las capacidades funcionales de un órgano como el cerebro. Es decir, los nińos desnutridos abandonaban prematuramente la escuela o debían repetir los primeros cursos, porque carecían de la capacidad intelectual adecuada para el aprendizaje.

El retraso cognitivo fue el correlato biológico del paradigma desarrollista que durante la segunda mitad del siglo xx observó a la infancia como la primera etapa del desarrollo humano e identificó sus formas de frustración. El informe elaborado en 1962 por una nueva comisión mixta de expertos norteamericanos y chilenos identificó que la deficiencia física - estimada en un io \% de déficit en peso y en estatura - se producía y era más aguda entre los 3 y 5 años de edad. A los 7 años de edad los niños eran físicamente subdesarrollados y muy susceptibles a enfermedades. Aunque esta se recuperaba relativamente entre los cinco y los nueve años, no llegaba a normalizarse (MONCKEBERG, 1967). Estos resultados dieron base científica al argumento político de que si el retraso escolar era debido a la desigualdad socioeconómica, la masificación de la escuela exigía resolver la segmentación del sistema educacional, que no solo reforzaba la desigual estructura social, sino que abortaba las oportunidades para superar el subdesarrollo. La premisa de que el proceso educativo era el medio de selección y ascenso social — por ende el factor decisivo en la transformación de la sociedad- expresó el dilema de priorizar entre políticas públicas y, dentro de la educacional, por niveles. En su informe, Chile no estaba tan mal respecto de los otros países de la región. En 1960, un 82,2 \% de los niños entre 7 y I4 ańos estaba matriculado en la escuela; sin embargo, el 7I,5 \% la había abandonado antes de completarla. Asimismo, el informe evaluó positivamente la mayor prevalencia de una relación adecuada entre la edad biológica y el nivel educacional entre los escolares. No obstante, este problema persistía, ya que el porcentaje de nińos entre 6 y 7 años de edad matriculados en primer año en I954 era similar al de 1962, lo que representaba un 30,I \% y 35,6 \% respectivamen$\mathrm{te}^{10}$. En estos primeros grados, además, la matrícula excedía a la población entre 7 y Io años de edad, por lo que se advierte que los alumnos rebasaban los límites etarios considerados normales para ese nivel. En síntesis, el carácter estructural del

10] Comisión de Planeamiento Integral de la Educación, Algunos Antecedentes para el Planeamiento Integral de la Educación chilena (1964). 
problema educacional contrastaba con la promesa de desarrollo como ampliación de las expectativas sociales y políticas.

La distinción entre el subdesarrollo del país como problema de crecimiento económico y el carácter socialmente desigual que dicho desarrollo pudo adquirir es necesaria para conocer cuáles fueron los elementos que allí se conjugaron. A nivel internacional, la masificación de la educación era una agenda urgente de integración y desarrollo tras la dramática experiencia de las guerras mundiales y las amenazas de proyectos de sociedad antagónicos a la democracia. En el contexto de la Guerra Fría, la escolarización masiva fue estimulada por los organismos internacionales que atribuyeron a la educación la capacidad de ampliar las bases del consenso social. Nuevos actores internacionales y sus proyectos desarrollistas a nivel global se entrelazaron a las políticas locales. El más significativo a nivel regional, por su peso político y los recursos financieros comprometidos, fue la Alianza para el Progreso del gobierno norteamericano. La meta era alcanzar el piso mínimo de 6 años de educación para todo niño latinoamericano en $1970^{11}$. El propósito se enmarcó en un ideario regional de cohesión social expresado en la Conferencia sobre Educación en Latinoamérica realizada en Santiago en 1962. Si bien el problema educacional fue un elemento central a una más amplia narrativa que acusaba elevadas tasas de analfabetismo, de mortalidad infantil, que identificaba los desafíos sociopolíticos de la masiva inmigración rural, la aguda urbanización y la pobreza popular, el debate a nivel nacional y latinoamericano no inauguró reformas políticas que podrían haber convertido a la escuela en canal de movilidad social. Cada país debía operar una transformación social correspondiente al nivel de educación necesario para la industrialización y el progreso económico. Pero no fue evidente qué lugar debía corresponder a la educación dentro de las políticas públicas ni su prioridad como ámbito de inversión económica para enfrentar la marginación social y espacial de amplios sectores de la población (WECKMANN, 1962). Chile requería, y la reforma de 1964 así lo expuso, un sistema educacional integrado que hiciera efectiva una enseñanza obligatoria de ocho años. Sin embargo, no hubo una inversión sustanciosa para ampliar el servicio educacional y los recursos se concentraron en el nivel secundario y técnico en función del plan de desarrollo nacional (HAMUY, 1960). Tampoco hubo inversión social en

11] Carta de Punta del Este de 1961: Plan Decenal de Educación de la Alianza para el Progreso. 
educación. Si bien los niños adquirieron cierta prioridad en la política pública conceptualizados como capital humano, la concreción de una política social en salud y alimentación escolar significó unas reducidas posibilidades de enfrentar la desnutrición. Aunque la escuela fue percibida como vía de salida a la pobreza, tanto nacional como individual, paradójicamente, los modos de implementar la asistencia escolar frustraron sus intenciones originales.

\section{EL SENTIDO COHESIONADOR DEL BIENESTAR}

Los modos para densificar el entretejido social comprendido desde el horizonte de una sociedad inclusiva no podían ser aquellos que la habían segmentado. El mayor y más complejo conocimiento e involucramiento de una pluralidad de especialistas en educación, economía, sociología y ciencia política develaron que la educación formaba el capital vital de un país, redituando enormemente en su desarrollo socioeconómico. Pero, al mismo tiempo, estos saberes identificaron que el rendimiento social de la educación variaba según el nivel general de desarrollo previo. Es decir, el nivel educacional era resultado y causa del nivel socioeconómico del país ${ }^{12}$. Por tanto, la función de la educación en el desarrollo requería otra fuerza en la dirección inversa ${ }^{13}$. Ese fue el tenor del debate suscitado a raíz de las tensiones y contradicciones manifestadas por el proceso de escolarización masiva. La discusión pública replanteó con vigor cuál era el sentido de la educación, al menos para el Estado, ya no como la necesidad decimonónica de orden social, sino como justicia individual. Aunque la pobreza, explicitó la Ley de Obligatoriedad Escolar, no eximía cumplir con la escuela, tampoco comprometió la ayuda estatal para resolver la imposibilidad material por parte de los hogares populares de matricular a sus nińos. Dado el contexto socioeconómico tan vulnerable que afectaba

12] El problema era latinoamericano: la población adulta presentaba un nivel educativo promedio de tan solo 2,2 años de escolaridad y el $40 \%$ era analfabeta. Un $68 \%$ (38 180000 niños) del total de población entre 7 y 14 años estaba matriculado en la escuela y, de aquellos que iniciaban la primaria, solo el $17 \%$ la terminaba.

13] Estudios desde la historia de la educación, por un lado, y economía de la educación, por otro, han discutido con evidencia contundente dicha relación evidenciando casos en que la escolarización no ha redundado en mayor crecimiento económico y también ha reproducido la desigualdad existente (BOURDIEU, 1997; MAYNES, 1985; VAUGHAN, 1982). 
irremediablemente al desarrollo del niño, como reiteradamente insistieron los directores de escuela y profesores, se requería algo más que la escuela para atraerlo.

¿Cómo podrían los niños participar efectivamente del proceso de escolarización si no eran físicamente aptos para el aprendizaje? Esta preocupación, constantemente planteada en la Revista de Educación, apeló al imperativo social del Estado y la necesidad de ampliar sus ámbitos de intervención para garantizar el mínimo de bienestar necesario para el desarrollo de una vida digna consagrado por la Constitución Política de 1925. Las limitaciones a la expansión social de la escuela persistían con fuerza y demostraban que la gratuidad de la educación era un principio antes que una realidad y que la escuela no constituía la red material de servicios que los niños necesitaban. En primer lugar, los niños estaban desnutridos antes de matricularse en la primaria, a pesar del incipiente sistema de bienestar diseñado por la legislación laboral de i924 y codificada en 1931. Los esfuerzos estatales concretados por la Ley de Medicina Preventiva en el gobierno de Aguirre Cerda permitieron extender a partir del año 1938 la atención en salud a todas las madres — no solo a las trabajadoras - durante el embarazo y el parto, además de la provisión de leche para los nacidos hasta cumplir los 2 años de edad (ZÁrATE, 2008). Esta incipiente red de bienestar, aunque precaria, pudo revertir en parte la condición desnutrida de los niños dentro de su radio de acción. Aquellos de las poblaciones obreras en áreas controladas por un hospital, el cual además administraba consultorios periféricos, tenían acceso a centros de atención materno e infantil. Dada cierta infraestructura existente, también estas zonas eran asistidas por otras instituciones particulares. Hubo avances significativos, aunque socialmente segmentados, lo que generó bolsones de miseria. La mortalidad infantil disminuyó y la desnutrición solo afectó, en un grado leve, al I 5 \% de los preescolares. Pero no ocurrió lo mismo en otras zonas urbanas sobrepobladas, que concentraban la mano de obra fabril y no contaban con establecimientos de salud (ARIZTía, 1967).

En segundo lugar, los infantes, tras cumplir dos años de edad, carecieron de un espacio de protección alimenticia durante el periodo preescolar. La opción focalizada hacia los niños más pequeños presupuso que más tarde los alimentaría la escuela a través del desayuno. Sin embargo, los niños entre los tres y cinco años de edad quedaron desamparados. El nivel parvulario como base del sistema educacional era muy incipiente y demoraría varias décadas en expandirse. Los establecimientos fiscales de párvulos eran escasos y la cantidad de niños que ingresaron a estos representó el $0,5 \%$ y el 3,3\% del total de la matrícula primaria 
entre 1932 y 1957, respectivamente. En consecuencia, la mayoría de los niños que llegaron a la escuela ya estaban desnutridos.

La política asistencial hacia el escolar no pudo ser efectiva. Los programas de alimentación no contaron con un programa universalmente comprehensivo y los modos de implementar el auxilio a través de corporaciones públicas a nivel municipal fueron inoperantes. La regulación de la obligatoriedad escolar había creado en 1929 las Juntas Comunales de Auxilio con el fin de vigilar el cumplimiento de la ley y supervisar los servicios de alimentación que debían instalarse. En este sentido, los servicios de alimentación escolar fueron un componente constitutivo del Estado de bienestar y de la democracia social, no un resultado o consecuencia (RENGIFO, 20I8). Institucionalmente, estas juntas significaban un avance respecto de la beneficencia pública que hasta entonces realizaba una labor por sobre sus capacidades y recursos, pero su funcionamiento fue parcial. Una década demoró conseguir los medios económicos para financiar el desayuno en las escuelas, cuya inversión sobrepasaba a los cortos aportes municipales. Si bien el Estado incrementó sustanciosamente su rol en el financiamiento, administración y directa provisión de estos servicios sociales, recién en 1953 las Juntas contaron con una estructura nacional que las coordinara y apoyara efectivamente. La transformación de este programa de bienestar estuvo en el corazón de la reforma de i964 que creó la Junta Nacional de Auxilio Escolar y Becas — pieza crucial de la reforma social y política para enfrentar la crisis_-, lo que posibilitó la satisfacción de al menos las carencias alimenticias elementales.

Tanto la precaria institucionalidad descrita como sus reducidos recursos implicaron que el auxilio escolar fuera selectivo entre los escolares que recibieron protección social. Los niños privilegiados lo fueron por su condición de indigente. Esta calidad era evaluada por los agentes de salud y autoridades escolares, debiendo además concurrir que el escolar asistiera regularmente a la escuela. Dado este requisito, cuestionado por las estadísticas que mostraban la relación negativa entre pobreza y asistencia, el auxilio escolar dificultosamente pudo llegar a los más pobres. Estimando a partir de los registros recogidos por la Junta, i de cada 20 niños matriculados en la primaria recibió al menos un vaso de leche como desayuno y otro número menor también obtuvo almuerzo ${ }^{14}$.

14] Memorias de la Junta Nacional de Auxilio Escolar. 
La expansión social de la escuela fue tangencial al régimen de bienestar desarrollado durante este periodo. La relación entre ambos procesos revela las temporalidades variadas de las políticas públicas. Teniendo en cuenta que, en el caso chileno, la masificación de la escuela difícilmente podría explicarse de un modo comprehensivo sin considerarla un proyecto estatal, cuya aparente evidencia no exime de la necesidad de especificar ese interés (LINDQUIST-WONG, I995; FULLER Y RUBINSON, 1992). Las políticas educacionales se entrelazaron a la provisión de prestaciones básicas en salud y previsión mediante el sistema de seguridad social. Los niños favorecidos por este incipiente sistema fueron privilegiados entre la población infantil. Por un lado, ellos fueron sujetos de dicha política social como hijos de los cotizantes. Ser carga familiar de un trabajador era una opción voluntaria del progenitor, quien debía inscribir a sus hijos en el sistema cotizando un porcentaje adicional por ellos. Ese costo era muy alto para quienes consumían todos sus recursos en la sobrevivencia del hogar. Por otro lado, los seguros sociales distinguieron entre obreros y empleados, entre el sector público y el privado, repercutiendo estas diferencias en las condiciones, requisitos de acceso a las prestaciones y calidad de los beneficios entregados por el sistema. La mayoría de los trabajadores del país ingresó a la Caja de Seguro Obligatorio, entidad estatal correspondiente al régimen general y la fiscalizadora del sistema. Este numeroso sector obrero era también el de menores salarios en contraste con los mejores sueldos de una minoría de empleados públicos y particulares cotizantes en varias otras administradoras de seguros (RENGIFO, 2017 y 20I6). En prácticamente todos los aspectos anteriores hubo reformas legales tendientes a incorporar un mayor número de personas a la seguridad social; sin embargo, el criterio prevaleciente fue la integración diferenciada. Con todo, el sistema de seguridad social protegió indirectamente a los escolares a través de programas particulares y de servicios públicos de carácter universal, alcanzando a un tercio de la población infantil según los cálculos disponibles para la década de I950 (BAEZA, 1950).

\section{CONCLUSIONES}

La masificación de la educación fue tardía porque la propia escuela perpetuó la desigualdad social. Si bien la mayoría de la población chilena cursó un par de años en la primaria, la expansión educacional representó una base extensa 
pero delgada debido a una desigualdad estructural a la sociedad chilena que las políticas educacionales no pudieron, o no quisieron, alterar. Desde la perspectiva educacional, las amenazas de quiebre social eran representadas por amplios sectores de población semialfabetos y los esfuerzos por masificar la educación básica develaron las tensiones entre integración, marginación y segregación social. El análisis de los ritmos del proceso de escolarización a la luz de las políticas educacionales del periodo constata que el problema educacional era biológico, porque la desnutrición padecida por los niños afectaba irreversiblemente en sus mentes discapacitadas para el aprendizaje. Los desafíos del período eran no solo masificar la matrícula primaria, sino que alcanzar la correspondencia entre la edad biológica y el nivel educacional. Sin desmerecer los esfuerzos estatales como estímulos al incremento de la escolarización, estos no fueron preponderantes. Tanto el sistema educacional como el régimen de bienestar fueron inclusivos, pero segmentados e inequitativos. Los niños abandonaban prematuramente la escuela y no continuaban estudiando, porque también este sistema carecía de una estructura orgánica, es decir, sus distintos niveles eran estratos sobrepuestos casi sin relación entre sí. En este contexto, la articulación de experiencias, nuevos conocimientos y el discurso democratizador sobre los vínculos sociales a través de la política de escolarización desestabiliza la premisa funcionalista de que la expansión de la educación equivale a mayor cohesión social en términos de integración y desarrollo. La formulación e implementación de las políticas contuvieron una dimensión epistemológica que acompañó a las reapropiaciones por parte de los actores e instituciones de disciplinas como la biología y la sociología que permitieron repensar la relación entre cohesión social y pobreza.

El proceso de escolarización fue expansivo en una dirección que no necesariamente implicó cohesión social. Las políticas de escolarización tuvieron éxito en alcanzar a la mayoría de la población y en conservar a aquellos niños que ya estaban incorporados al sistema educacional, pero no a los itinerantes condicionados por su pobreza. Por un lado, el sentido inclusivo de la escuela no necesariamente fue equitativo, sino que se tradujo en unas políticas segmentadas espacial y socialmente. La arquitectura institucional observada desde la demografía escolar ilumina el proceso de escolarización también como contracara al proyecto desarrollista de cohesión social. La educación, formuló, promovería la movilidad social pero ello requería generar un cierto piso demográfico, porque la relación entre sociedad y desarrollo era íntima. Por otro lado, las opciones políticas tomadas con el fin 
de expandir socialmente la escuela mantuvieron unas amarras y soltaron otras, dificultando severamente que los establecimientos educacionales fueran una plataforma efectiva de asistencia escolar contra la pobreza infantil. Las posibilidades de protección que ofreció fueron mediante los servicios de alimentación que seleccionaron por indigencia y, eventualmente, a través de programas de salud y beneficios económicos de la seguridad social.

Las soluciones vislumbradas apuntaron a la función primordial del Estado en expandir la educación, debiendo asegurar la gratuidad por lo menos de la primaria. Los esfuerzos de reforma persiguieron resolver las fallas de la administración escolar expresadas en la diferente duración de la escolaridad primaria entre zonas rurales y urbanas; enfrentar de esta forma las carencias pedagógicas que se reflejaban en el escaso rendimiento de las escuelas mayoritariamente correspondientes a aquellas ubicadas en barrios pobres y zonas rurales. La crisis social, económica y política con que termina el periodo contuvo también esa fractura nacional que los procesos de escolarización y democratización pudieron acortar solo en parte. Los postulados de la reforma educacional insistieron en que la escuela estaba en el corazón de la sociedad y, por ende, el cambio social que haría posible el desarrollo nacional debía comenzar en ella. En consecuencia, el planeamiento de la educación bajo el gobierno de Frei Montalva pretendió ser un plan integral que garantizara la permanencia y continuidad de los niños en el sistema educacional. A pesar de la persistente desigualdad de ingresos en Chile, en el mediano plazo, los esfuerzos en salud y alimentación permitieron elevar los estándares de vida atribuible al desarrollo institucional e infraestructural del primer piso de prestaciones sociales. Las políticas del periodo lograron satisfacer importantes necesidades básicas entre la población que impactaron en el descenso de la tasa de mortalidad infantil disminuyeron entre 1965 y 1975 de $97,3 \%$ a 57,6 \%- y en el incremento de la expectativa de vida al nacer (MCGUIRE, 20IO; THROP, I998). Durante la segunda mitad del siglo xx, Chile registró un mejoramiento de las condiciones de salud, sin embargo, permanecía un grave déficit biológico entre los sectores sociales más pobres. Si bien el porcentaje de menores de seis años de edad que sufrían algún grado de desnutrición disminuyó de 36,9\% a 19,5\% entre 1960 y 1970, todavía un quinto de la población infantil ingresaba a la escuela fisiológicamente trastornado. Sus efectos en la democratización profunda de la cobertura escolar serán percibidos a fines del siglo xx. 


\section{Referencias bibliográficas}

Anuario Estadístico de la República de Chile, años 1890 a 1957.

ARIZTÍA, ANÍBAL (1967): «El hambre o desnutrición en la población de Chile», en: Revista Chilena de Pediatría, nº 28.

BAEZA, ARTURO (1950): «Pediatría y Seguridad Social», en: Revista Chilena de Pediatría, vol. $1, \mathrm{n}^{\circ} 21$.

BARBA, CARLOS Y COHEN, NÉSTOR (2011): «Introducción. Hacia una visión crítica de la cohesión social en América Latina», en: Barba, Carlos y Cohen, Néstor (coords.), Perspectivas críticas sobre la cohesión social. Desigualdad y tentativas fallidas de integración social en América Latina, Buenos Aires, Consejo Latinoamericano de Ciencias Sociales.

MINISTERIO DE EDUCACIÓN (1961): Bases generales para el planeamiento integral de la educación chilena, Santiago.

BEHAGG, CLIVE (1997): «Myths of Cohesion: Capital and Compromise in the Historiography of Nineteenth-Century Birmingham», en: Social History, vol. 3, no 11, pp. 375-384.

BENAVOT, AARON (1997): «Institutional approach to the Study of Education», en: Lawrence Saha (ed.), International Encyclopedia of the Sociology of Education, Oxford: Elsevier Science.

BOURDIEU, PIERRE (1997): Capital cultural, escuela y espacio social, México, Siglo XXI Editores.

CARTA DE PUNTA DEL ESTE DE 1961: Plan Decenal de Educación de la Alianza para el Progreso.

CASTORIADIS, CORNELIUS (1993): La institución imaginaria de la sociedad, Buenos Aires, Tusquets. COMISIÓN DE PLANEAMIENTO INTEGRAL DE LA EDUCACIÓN, MINISTERIO DE EDUCACIÓN PÚBLICA DE CHILE (1964): Algunos Antecedentes para el Planeamiento Integral de la Educación chilena. Santiago, Editorial Universitaria.
COX, CRISTIÁN Y SCHWARTZMAN, SIMON (2009): Políticas Educativas y Cohesión Social en América Latina, Uqbar Editores.

CUELLAR, ROBERTO (2010): Cohesión social y democracia, International Institute for Democracy and Electoral Assistance.

DIRECCIÓN GENERAL DE ESTADÍSTICA (1931): Resultados del X Censo de la Población efectuado el 27 de noviembre de 1930 y estadísticas comparativas con Censos anteriores, Santiago, Imprenta Universo.

DIRECCIÓN GENERAL DE ESTADÍSTICA (1933): Censo educacional de 1933, Santiago.

DIRECCIÓN GENERAL DE ESTADÍSTICA (1940): XI Censo Nacional de Población de Chile realizado el día 28 de noviembre de 1940, Santiago.

DRAGONI, CARLO Y BURNET, ETIENNE (1939): «L'Alimentation Populaire au Chili. Première en-quete générale de 1935. Rapport rédigé par le Prof. Carlo Dragoni avec la coopération du Doct. Et. Burnet. Première enquête Générale de 1935», en: Revista de Medicina e Higiene Preventiva, 10-12, pp. 410-611.

DUARTE, A. (1943): «Sanidad Escolar Rural», en: Revista Chilena de Pediatría, vol. 5, no 14, pp. 348-355.

DURKHEIM, EMILE (1967): De la división del trabajo social, Schapire:, Universidad Complutense de Madrid.

EGAÑA, MARÍA L. (1995): «La cobertura de la educación primaria popular en Chile: 1890-1920», en: Boletín de Investigación Educativa, n 10, pp. 308-319.

FULLER, BRUCEY RUBINSON, RICHARD (EDS.) (1992): The Political Construction of Education: The State, School Expansion, and Economic Change, New York, Praeger.

GRASSAU, ERIKA Y ORELLANA, EGIDIO (1959): «Desar- 
rollo de la educación chilena desde 1940», en: Boletín Estadístico de la Universidad de Chile III, n ${ }^{\circ} 1$, Santiago: Instituto de Investigaciones Pedagógicas Sección Estadística.

HAMUY, EDUARDO (1960): Educación elemental, analfabetismo y desarrollo económico, Santiago, Editorial Universitaria.

HUNTINGTON, SAMUEL P. (1991): The Third Wave: Democratization in the Late Twentieth Century, University of Oklahoma Press.

ILLANES, MARÍA A. (1990): Ausente, señorita: el niño chileno, la escuela para pobres y el auxilio 1890/1990: hacia una historia social del siglo XX en Chile, Santiago: Junta Nacional de Auxilio Escolar y Becas.

JUNTA NACIONAL DE AUXILIO ESCOLAR (1959): Memoria anual. Labor desarrollada 1953-1959, Santiago. KOSELLECK, REINHART (2012): Historias de conceptos. Estudios sobre semántica y pragmática del lenguaje político y social, Madrid, Trotta.

LABARCA, AMANDA (1944): Bases para una política educacional, Buenos Aires, Losada.

LABARCA, AMANDA (1939): Historia de la enseñanza en Chile, Santiago: Publicaciones de la Universidad de Chile.

LINDQUIST-WONG, PIA (1995): «Constructing a Public Popular Education in Sao Paulo, Brazil», en: Comparative Education Review, vol. 1, no 39, pp. 120-141.

MAYNES, MARY JO (1985): Schooling in Western Europe, Albany, State University of New York Press. MCGUIRE, JAMES (2010): «Politics, Policy, and Mortality Decline in Chile, 1960-1995», en: Salvatore, Ricardo (Eds), Living Standards in Latin American History. Height, Welfare, and Development 17502000, Massachusetts, Harvard University Press. MARSHALL, THOMAS H. (1950): Citizenship and Social Class: And Other Essays, Cambridge University Press.
MEYER, JOHN W.; RAMÍREZ, FRANCISCO O. Y NUHOGLU, YASEMIN S. (1992): «World Expansion of Mass Education, 1870-1980", en: Sociology of Education, vol. 2, n 65, pp. 128-149.

MINISTERIO DE EDUCACIÓN (1961): Bases generales para el planeamiento integral de la educación chilena, Santiago.

MINISTERIO DE EDUCACIÓN Revista de Educación números de 1934 a 1944.

MONCKEBERG, FERNANDO (1967): «Estudio del estado nutritivo y de las condiciones de vida de la población infantil en la provincia de Curicó», en: Revista Chilena de Pediatría, n 38, 491-535. O'DONNELL, GUILLERMO (1999): «Pobreza y desigualdad en América Latina: algunas reflexiones políticas», en: O’Donnell, Guillermo y Tokman, Víctor (comps.), Pobreza y desigualdad en América Latina. Temas y nuevos desafíos, Buenos Aires, Paidós.

PARRAD0, EMILIO A. (1998): «Expansion of Schooling, Economic Growth, and Regional Inequalities in Argentina», en: Comparative Education Review, vol. 3, no 42, pp. 338-364.

POBLETE, MOISÉS (1942): El standard de vida de las poblaciones de América, Santiago, Prensas de la Universidad de Chile.

PONCE DE LEÓN, MACARENA (2018): «Los dilemas del crecimiento de la educación», en: Serrano, Sol; Ponce de León, Macarena; Rengifo, Francisca y Mayorga, Rodrigo (eds.), Historia de la educación en Chile. Tomo III: Democracia, exclusión y crisis (1930-1964), Santiago, Taurus Random House, pp. 63-92.

PONCE DE LEÓN, MACARENA (2010): «La llegada de la escuela y la llegada a la escuela: La extensión de la educación primaria en Chile, 1840-1907», en: Historia, vol. 2, n 43, pp. 449-486.

RAMÍREZ, FRANCISCO 0. Y BOLI, JOHN (1987): «The Political Construction of Mass Schooling: European 
Origins and Worldwide Institutionalization», en: Sociology of Education, vol. 1, n 60, pp. 2-17. RENGIF0, FRANCISCA (2016): «El significado político de la familia en la institucionalización del Estado de seguridad social chileno, 1920-1930», en: Revista de Estudios Avanzados, n² 26, pp. 56-75. RENGIF0, FRANCISCA (2017): «Desigualdad e inclusión. La ruta del estado de seguridad social chileno, 1920-1970", en: Hispanic American Historical Review, vol. 3, n 97, pp. 485-521. RENGIF0, FRANCISCA (2018): «El enemigo del niño es el hambre», en: Serrano, Sol; Ponce de León, Macarena; Rengifo, Francisca y Mayorga, Rodrigo (eds.), Historia de la educación en Chile. Tomo III: Democracia, exclusión y crisis (1930-1960), Santiago, Taurus Random House, pp. 157-208. REPÚBLICA DE CHILE. Dirección General de Estadística (1895): Censo de población de la República de Chile, Santiago.

REPÚBLICA DE CHILE (1907): Censo de población de la República de Chile levantado en 1907, Santiago.

REPÚBLICA DE CHILE (1925): Censo de población de la República de Chile levantado el 15 de diciembre de 1920, Santiago: Sociedad Imprenta y Litografía Universo.

REPÚBLICA DE CHILE (1933): Censo de educación. ROSEMBLATT, KARIN A. (2001): "Charity, Rights, and Entitlement: Gender, Labor, and Welfare in EarlyTwentieth-Century Chile», en: Hispanic American Historical Review, vol. 3-4, no 81, pp. 555-585. ROSANVALLON, PIERRE (1995): La nueva cuestión social. Repensar el Estado Providencia, Buenos Aires, Ediciones Manantial.

ROSANVALLON, PIERRE (2011): Democratic Legitimacy-Impartiality, Reflexivity, Proximity, Princeton University Press.

SALAS, DARÍ0 (1917): El problema educacional. Bases para la construcción de nuestro sistema escolar primario, Santiago, Imprenta y Litografía Universo.

SCHTEINGART, MARI0 (1935): Alimentación y dietética en Buenos Aires, Aniceto López editor.

SERVICIO NACIONAL DE ESTADÍSTICA Y CENSOS (19561958): XII Censo general de población y de vivienda levantado el 24 de abril de 1952, Santiago, Gutemberg.

SERVICIO NACIONAL DE ESTADÍSTICA Y CENSOS (1964): Población del país: Características básicas de la población (Censo de 1960), Santiago.

SOIFER, DAVID (2009): «The Sources of Infraestructural Power. Evidence from Nineteenth-Century Chilean Education", en: Latin American Research Review, vol. 2, no 44, pp. 158-180.

SORJ, BERNARDO Y TIRONI, EUGENIO (2007): Cohesión social en América Latina: un marco de investigación, en: Pensamiento Iberoamericano $n^{\circ} 1$, pp. 105-127.

THORP, ROSEMARY (1998): Progreso, pobreza y exclusión: Una historia económica de América Latina en e/ sig/o XX, Banco Interamericano de Desarrollo.

TIRONI, EUGENIO; MELLER, PATRICIO; PEÑA, CARLOS ET AL. (2008): Cohesión Social en América Latina. Redes, Estado y Mercado. Soportes de la cohesión social latinoamericana, Uqbar.

TORRES, CARLOS A. Y PUIGGRÓS, ADRIANA (1995): «The State and Public Education in Latin America», Comparative Education Review, vol. 39, n 1 , pp. 1-27.

TORRES, ISABEL (2014): La crisis del sistema democrático: las elecciones presidenciales y los proyectos políticos excluyentes. Chile 1958-1970, Santiago, Editorial Universitaria, Centro de Investigaciones Diego Barros Arana. URZÚA, GERMÁN (1992): Historia política de Chile y su evolución electoral desde 1810 a 1992, Santiago: Editorial Jurídica de Chile. 
VALENZUELA, SAMUEL (1985): Democratización vía WECKMANN, LUIS (1962): «La conferencia sobre edureforma. La expansión del sufragio en Chile, cación en Latinoamérica», en: Foro Internacional Buenos Aires, Instituto de Desarrollo Económico (Colegio de México), nº 1, vol. 3, pp. 83-103. y Social. ZÁRATE, MARÍA S. (2008): «Las madres obreras y el VAUGHAN, MARY K. (1982): The State, Education, Estado chileno. La Caja del Seguro Obligatorio, and Social Class in Mexico, 1880-1928, DeKalb, 1900-1950", en: Montecino, Sonia (comp.), Northern Illinois University Press.

Mujeres chilenas, fragmentos de una historia, Santiago, Catalonia. 